\title{
Pleiotropic Effects of Tetracyclines in the Management of COVID-19: Emerging Perspectives
}

OPEN ACCESS

Edited by:

Robert Wallace Malone, Independent Researcher, Madison, VA, United States

Reviewed by: Harmanjit Singh,

Government Medical College and Hospital, India

Piero Sestili,

University of Urbino Carlo Bo, Italy

*Correspondence:

Natália Cruz-Martins ncmartins@med.up.pt Gaber El-Saber Batiha gaberbatiha@gmail.com

Specialty section: This article was submitted to Respiratory Pharmacology, a section of the journal Frontiers in Pharmacology

Received: 16 December 2020 Accepted: 18 January 2021

Published: 23 April 2021

Citation: Al-kuraishy HM, Al-Gareeb Al, Alqarni M, Cruz-Martins $N$ and El-Saber Batiha G (2021) Pleiotropic

Effects of Tetracyclines in the Management of COVID-19:

Emerging Perspectives.

Front. Pharmacol. 12:642822. doi: 10.3389/fphar.2021.642822

\author{
Hayder M. Al-kuraishy ${ }^{1}$, Ali I. Al-Gareeb ${ }^{1}$, Mohammed Alqarni ${ }^{2}$, Natália Cruz-Martins ${ }^{3,4,5 *}$ \\ and Gaber El-Saber Batiha ${ }^{6 *}$
}

${ }^{1}$ Department of Clinical Pharmacology and Medicine, College of Medicine, Al-Mustansiriya University, Baghdadlraq, ${ }^{2}$ Department of Pharmaceutical Chemistry, College of Pharmacy, Taif University, Taif, Saudi Arabia, ${ }^{3}$ Faculty of Medicine, University of Porto, Porto, Portugal, ${ }^{4}$ Institute for Research and Innovation in Health (i3S), University of Porto, Porto, Portugal, ${ }^{5}$ Laboratory of Neuropsychophysiology, Faculty of Psychology and Education Sciences, University of Porto, Porto, Portugal, ${ }^{6}$ Department of Pharmacology and Therapeutics, Faculty of Veterinary Medicine, Damanhour University, Damanhour, Egypt

Coronavirus disease 2019 (COVID-19) is a global infectious disease caused by severe acute respiratory syndrome coronavirus 2 (SARS-CoV-2). Approximately 15\% of severe cases require an intensive care unit (ICU) admission and mechanical ventilation due to development of acute respiratory distress syndrome (ARDS). Tetracyclines (TCs) are a group of bacteriostatic antibiotics, like tetracycline, minocycline, and doxycycline, effective against aerobic and anaerobic bacteria as well as Gram-positive and Gram-negative bacteria. Based on available evidences, TCs may be effective against coronaviruses and thus useful to treat COVID-19. Thus, this review aims to provide a brief overview on the uses of TCs for COVID-19 management. SARS-CoV-2 and other coronaviruses depend mainly on the matrix metalloproteinases (MMPs) for their proliferation, cell adhesion, and infiltration. The anti-inflammatory mechanisms of TCs are linked to different pathways. Briefly, TCs inhibit mitochondrial cytochrome $c$ and caspase pathway with improvement of lymphopenia in early COVID-19. Specifically, minocycline is effective in reducing COVID19-related complications, through attenuation of cytokine storm as apparent by reduction of interleukin (IL)-6, IL-1, and tumor necrosis factor (TNF)- $\alpha$. Different clinical trials recommend the replacement of azithromycin by minocycline in the management of COVID-19 patients at high risk due to two main reasons: 1) minocycline does not prolong the QT interval and even inhibits ischemia-induced arrhythmia; 2) minocycline displays synergistic effect with chloroquine against SARS-CoV-2. Taken together, the data presented here show that TCs, mainly doxycycline or minocycline, may be potential partners in COVID-19 management, derived pneumonia, and related complications, such as acute lung injury (ALI) and ARDS.

Keywords: coronavirus disease 2019, acute respiratory distress syndrome, SARS-CoV-2, COVID-19, tetracyclines

\section{INTRODUCTION}

Coronavirus disease 2019 (COVID-19) is a global infectious disease, actually considered a pandemic by the WHO, caused by severe acute respiratory syndrome coronavirus 2 (SARS-CoV-2). SARS$\mathrm{CoV}-2$ is a positive-sense, single-strand RNA virus sharing a genetic similarity with other Betacoronaviruses, such as Middle East respiratory syndrome coronavirus 1 (MERS-CoV-1) and 
severe acute respiratory syndrome coronavirus 1 (SARS-CoV-1) (Al-Kuraishy et al., 2020a). Specifically, SARS-CoV-2 binds to a specific receptor, called angiotensin converting enzyme 2 (ACE2) receptors, which are highly expressed in lung epithelial cells, proximal renal tubules, the heart, and even the brain. ACE2 is considered as the main target of the virus. SARS-CoV-2 infection triggers an acute host immune response, inflammatory reaction, and cytokine storm, leading to acute lung injury (ALI) and acute respiratory distress syndrome (ARDS) (Al-kuraishy et al., 2020b).

The genomic analysis of SARS-CoV-2 shows four structural proteins, namely, nucleocapsid protein (NP), membrane protein (MP), envelop protein (EP), and spike protein (SP), and four nonstructural proteins, namely, 3-chymotrypsin-like proteins (3CLpro), papain-like protease (PLpro), helicase, and RNA polymerase (Ser et al., 2020). Both PLpro and 3CLpro are involved in SARS-CoV-2 replication and transcription, with these proteins' inhibition leading to a significant suppression of viral replications. However, 3CLpro is regarded as the main protease (Mpro), which concerns to all steps of SARS-CoV-2 life cycle. Thus, Mpro inhibitors may be effective against COVID-19 through inhibition of SARS-CoV-2 replication (Forster et al., 2020).

The clinical presentation and spectrum of COVID-19 varies from asymptomatic to mild-moderate clinical forms, with severe cases often needing hospitalization. Approximately $15 \%$ of severe cases require intensive care unit (ICU) admission and mechanical ventilation due to development of ARDS (Al-Kuraishy et al., 2020a). At present, different treatment modalities and approaches have been proposed to treat COVID-19, but there is no strong clinical evidence of their efficacy and safety, as they derive from animal and in vitro studies or from previous experiences during MERS-CoV and SARS-CoV pandemics. More recently, different randomized and nonrandomized clinical trials have been adopted; besides, prospective studies are underway (Trivedi et al., 2020).

Tetracyclines (TCs) are a group of bacteriostatic antibiotics, including tetracycline, minocycline, and doxycycline, which have shown to be effective against aerobic and anaerobic bacteria as well as Gram-positive and Gram-negative bacteria, with exceptions of Proteus species and Pseudomonas aeruginosa strains, which have intrinsic resistance. TCs act through inhibition of charged aminoacyl-tRNA attachment on the 30 subunits of microbial ribosomes (Peiris et al., 2017). Depending on present and previous evidences, TCs may be effective against coronaviruses, including SARS-CoV-2. Indeed, SARS-CoV-2 and other coronaviruses depend mainly on matrix metalloproteinases (MMPs) for their proliferation, cell adhesion, and infiltration. Zinc is a corner stone of MMPs; therefore, zinc chelation by TCs may limit SARS-CoV-2 infection and COVID-19 development (Ohe et al., 2020). Recent data have reported that TCs have noteworthy antiviral effects against single-strand, positive-sense RNA viruses, like dengue virus and SARS-CoV-2, through inhibition of RNA polymerase and serine protease (Li et al., 2017). In addition, doxycycline reduces viral replication, inhibiting the viral entry into cultured cells, with a consequent reduction of viral load (Mosquera-Sulbaran and HernándezFonseca, 2021). Indeed, a previous study revealed that the combination of doxycycline and ribavirin is more effective against chikungunya virus (Ferreira et al., 2019). In this sense, this review aims to provide a brief overview on the potential role of repurposing TCs in the management of COVID-19 that requires comprehensive evidences before their use in clinical practice.

\section{TETRACYCLINES AND COVID-19}

TCs have shown great potential for the management of COVID19 through inhibition of SARS-CoV-2-induced hyperinflammation and cytokine storm, since TCs downregulate the production of inflammatory cytokines, including interleukins (IL-6, IL-33, and IL-1 $\beta$ ) and tumor necrosis factor (TNF)- $\alpha$. Also, doxycycline inhibits the expression of CD26 and CD147, which are important entry points for SARS-CoV-2 (Ohe et al., 2020). A study conducted by Yates et al. (2020) involving four case series of high-risk COVID-19 patients who were placed on doxycycline therapy for 5-14 days illustrated clinical and radiological improvements following 14 days of doxycycline therapy. Despite the safety profile of doxycycline, the authors do not recommend the general use of this drug for treating COVID-19 patients, except if under direct supervision and monitoring by physician.

Bonzano et al. (2020) confirmed that administration of doxycycline $200 \mathrm{mg} /$ day in six COVID-19 patients with anosmia and other respiratory symptoms for 8 days led to rapid recovery of smell within 2 days, with the average time of $2.5 \pm 0.5$ days. This improvement was related to the modulation of ACE2 and CD147 expressions at the olfactory epithelium.

It has been shown that SARS-CoV-2-induced hyperinflammation is linked to mast cell proliferation and stimulation at the respiratory submucosa with subsequent release of histamine, IL-1, and IL-33. Also, TCs and their derivative inhibit proliferation with induction of mast cell apoptosis and activation of protein kinase C, ultimately inhibiting respiratory inflammation and cytokine storm (Alam et al., 2020). Moreover, both minocycline and doxycycline are effective in attenuating COVID-19-induced ARDS and cytokine storm (Mostafa, 2020). Similarly, by virtue of their lipophilic properties, TCs have a higher penetration to the basement membrane of alveolar cells and may also efficiently cross the SARS-CoV-2 envelope (Mostafa, 2020). Therefore, the use of TCs seems promising in the management of COVID-19-derived pneumonia due to their ability to inhibit SARS-CoV-2 replication and associated inflammatory reactions. Furthermore, TCs are safer than chloroquine and antiretroviral drugs that are commonly and initially used in the management of COVID-19 pneumonia (Poinas et al., 2020).

Zhao and Patankar (2020) also found that TCs and doxycycline are more effective than either chloroquine or doxycycline in inhibiting SARS-CoV-2 binding to the ACE2, as they are able to inactivate the viral receptor-binding domain. In the same way, Bharadwaj et al. (2020), in a molecular docking computational study, found that TCs are effective against SARSCoV-2 through inhibition of membrane protein (Mpro). Based 
on combinatorial molecular simulation analysis, doxycycline and minocycline revealed to be potent inhibitors of SARS-CoV-2 Mpro, and therefore can be used in combinational therapy against SARS-CoV-2 infection. Moreover, different in vitro studies illustrated that doxycycline has anti-SARS-CoV-2 activity with suppression of bacterial coinfections and associated inflammatory changes (Gendrot et al., 2020). Previous experimental studies showed that minocycline was effective against Japanese encephalitis virus through modulation of microglial activation, neural apoptosis, and viral replication (Mishra and Basu, 2008).

In addition, a recent retrospective multi-institutional cohort study illustrated that within a year, TC users have low risk of ARDS, shorter stay length in the ICU, and lower need for mechanical ventilation. Both doxycycline and minocycline and other TCs exert potent anti-inflammatory effects as they inhibit the proliferation of T-cells and inflammatory cytokines with subsequent suppression of the development of ARDS. This finding indicates the prophylactic role of TCs in the prevention of COVID-19-induced ARDS. Also, TCs downregulate the CD40 ligand on T-cells and prevent lung inflammation progression during COVID-19 pneumonia (Byrne et al., 2020). Alam et al. (2020), in an observational study, examined 89 patients with COVID-19 from March to May 2020 and showed that early treatment with doxycycline (10 mg/day) for 7 days in high-risk nonhospitalized COVID-19 patients was linked to an early clinical recovery and decreased hospitalization and mortality.

\section{ANTI-INFLAMMATORY EFFECTS OF TETRACYCLINES IN COVID-19}

As stated above, the anti-inflammatory mechanisms of TCs are linked to different pathways at both cellular and molecular levels. Briefly, TCs inhibit mitochondrial cytochrome $c$ and caspase- 1 pathway with improvement of lymphopenia in early COVID-19 cases (Moullan et al., 2015). In COVID-19, invasion of alveolar epithelial type II (AEC-II) cells by SARS-CoV-2 causes inflammatory reactions through activation of nuclear factor kappa-light-chain enhancer of the activated B-cell pathway (NFkB). The activated NFkB pathway during COVID-19 leads to AEC-II cell apoptosis with reduction of alveolar surfactant and downregulation of ACE2, while it prolongs neutrophil survival and accumulation (Hariharan et al., 2020). Also, the activated $\mathrm{NF \kappa B}$ pathway increases the differentiation and response of the pro-inflammatory macrophage phenotype, which per se causes cytokine storm and further NFKB activation in a vicious cycle (Carcaterra and Caruso, 2021). Kircheis et al. (2020) revealed that inhibition of the NFkB pathway during SARS-CoV-2 infection might be a potential target in attenuating the cytokine storm progression through inhibition of pro-inflammatory cytokines, such as IL-6, IL-1, TNF- $\alpha$, chemokines, and adhesion molecules. Different inhibitors of the NFKB pathway, such as dexamethasone and acetylsalicylic acid, are also able to reduce the cytokine storm in severe COVID-19 and other viral infections (Neufeldt et al., 2020). Also, it has been reported that the anti-inflammatory effect of TCs is related to their ability to suppress NFkB transcription factor, through inhibition of I $\mathrm{B} \alpha$ (IKK) activation and nuclear translocation of NFkB (Sun et al., 2015). In COVID-19, TCs downregulate the $\mathrm{NF \kappa B}$ pathway and other inflammatory signaling pathways, such as p38, ERK1, and MAPK pathways, with significant inhibition of SARS-CoV-2 main protease (Mosquera-Sulbaran and Hernández-Fonseca, 2021).

These changes prevent T-cell suppressor apoptosis, which ultimately averts the exacerbation of immune activations in late COVID-19 pneumonia. Moreover, the apoptosis inhibition may stop endothelial dysfunction-induced microthrombosis (Ferreira et al., 2020). Also, TCs preclude neutrophil migration, chemotaxis, release of pro-inflammatory cytokines, local oxidative stress, and vascular leakage. Thus, TCs may prevent COVID-19-induced ARDS (Byrne et al., 2020). Specifically, doxycycline inhibits inflammation-induced lymphangiogenesis through attenuation of vascular endothelial growth factor signaling, thus attenuating neovascularization-induced alveolar hemorrhage, alveolar collapse, and refractory hypoxemia in COVID-19 pneumonia (Connors and Levy, 2020).

On the other hand, Portnoy et al. (2020) found that activation of MMPs, mainly MMP-2 and MMP-9, by SARS$\mathrm{CoV}-2$ leads to the degradation of alveolar basement membrane. Briefly, MMPs are synthesized and released from macrophages and neutrophils involved in ALI (Ueland et al., 2020). In addition, it has been shown that macrophage activation and infiltration is associated with SARS-CoV-induced ALI. Also, COVID-19 pneumonia is associated with macrophage activation syndrome with elevation of IL-6 levels. Therefore, MMP inhibition by TCs might explain the beneficial role of such agents in controlling COVID-19-induced ARDS as evident by the reduction of IL-6 serum levels (McGonagle et al., 2020). Recent data have shown that minocycline is an effective drug, being able to reduce COVID-19-related complications through attenuation of cytokine storm as apparent by the reduction of IL-6, IL-1, and TNF- $\alpha$ (Singh et al., 2020). Previously, Di Caprio et al. (2015) reported that a low dose but not large-dose of doxycycline is effective in the inhibition of inflammatory reactions in chronic inflammatory disorders. Thus, based on this evidence, minocycline and doxycycline seem to be promising drugs for COVID-19 therapy; however, different preclinical and clinical studies are warranted before the final recommendation of minocycline for COVID-19 treatment, due to reduction of all steps of inflammation (Carcaterra and Caruso, 2021).

On the other hand, but also worthy of note is that as chloroquine is extensively used alone or in combination with azithromycin in the management of COVID-19-derived pneumonia, the risk of QT prolongation and arrhythmias is increased, mainly in males and elderly patients, which represent the main proportion of COVID-19 population (Diana et al., 2020). Moreover, Wang et al. (2020) disclosed that $44.4 \%$ of hospitalized COVID-19 patients and $16.7 \%$ of those in ICU have arrhythmias due to electrolyte disturbances, hypoxia, and use of chloroquine alone or in combination with azithromycin. Therefore, various clinical studies have 
recommended the replacement of azithromycin by minocycline in the management of high-risk COVID-19 patients for two main reasons: 1) Minocycline does not prolong the QT interval and even inhibits ischemia-induced arrhythmia; 2) minocycline has a synergistic effect with chloroquine against SARS-CoV-2 (Diana et al., 2020). Therefore, early treatment with TCs, mainly doxycycline or minocycline, for high-risk COVID-19 patients seems linked to an early recovery, reduced hospitalization, and decreased mortality rate. Oliveira et al. (2020), in a clinical trial study, demonstrated that lopinavir plus doxycycline is more effective in the management of COVID-19 patients due to a synergistic inhibition of SARS-CoV-2 protease. Nevertheless, Cag et al. (2020) showed that combination of minocycline and chloroquine offers many benefits in the management of moderate-to-severe COVID-19 patients, since both drugs are widely available and cheap, and their contraindications are well-known and recognized. As well, the anticytokine and antimicrobial effects of this combination may mitigate both morbidity and mortality rates among COVID-19 patients alternative to the costlier drugs.

Regarding other modalities in the management of severe COVID-19 patients, tocilizumab (IL-6 antagonist) has been used to overcome the cytokine storm and development of ALI and ARDS. However, there are limited real-life data regarding the effect of tocilizumab in COVID-19 management (Gautam et al., 2020). A retrospective study showed that tocilizumab therapy may reduce the need for mechanical ventilation and death in patients with severe COVID-19 (Luo et al., 2020). However, Guaraldi et al. (2020) confirmed that tocilizumab was not effective in preventing intubation or death in hospitalized

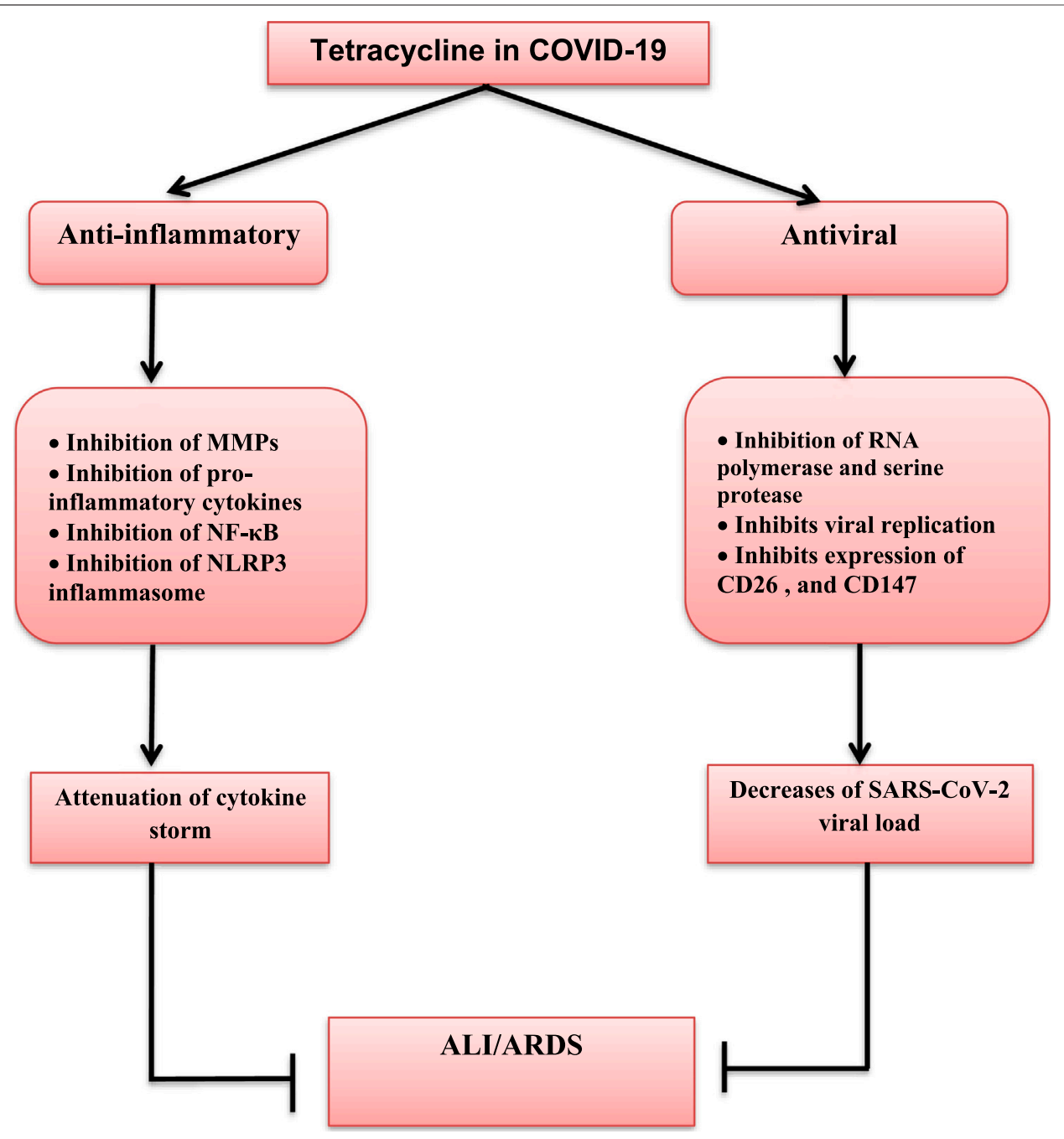

FIGURE 1 | Pleiotropic effects of tetracycline in SARS-CoV-2 infection. Tetracycline has antiviral and anti-inflammatory effects. Antiviral effects of tetracycline are through inhibition of RNA-polymerase and serine protease dependent viral replications, and inhibition of the expression of CD26 and CD147, which are regarded as entry-point for SARS-CoV-2 with subsequent reduction in viral load. Anti-inflammatory effects of tetracycline are through inhibition of matrix metalloproteinases (MMPs), nuclear factor kappa B (NF-kB), Nod-like receptor pyrin 3 (NLRP3) inflammasome, and release of pro-inflammatory cytokines with subsequent attenuation of cytokine storm development. Taken together, both anti-inflammatory and antiviral effects of tetracycline inhibit development of acute lung injury (ALI) and acute respiratory distress syndrome (ARDS) in COVID-19. 
COVID-19 patients. Besides, unlike TCs, tocilizumab is an expensive drug that also needs close monitoring during its parenteral administration since it may cause serious anaphylactic reactions, liver injury, and secondary bacterial infections (Stone et al., 2020). On the other hand, TCs are cheap, safe, and effective orally, not needing close monitoring and neither increasing the risk of bacterial coinfection; therefore, TCs are more effective than tocilizumab in the management of COVID-19 (Gatti et al., 2020).

Moreover, TCs could be helpful in another therapeutic situation rarely occurring in COVID-19 patients, that is, the treatment of bacterial superinfections. A recent report showed that superinfections sustained by atypical bacteria, such as Mycoplasma intracellular pathogens, seem to be the most frequent ones (Huang et al., 2020). Incidentally, TCs similar to azithromycin but with no cardiological issues are highly active against these bacteria which, on the contrary, are naturally insensitive to beta-lactams (Sodhi and Etminan, 2020; McKenna, 2020). This, along with their broad spectrum, could suggest using TCs as a treatment eventually in association with beta-lactams or other antibiotics in the empirical management of superinfections (Conforti et al., 2020). Also, an indiscriminate use of TCs in the absence of bacterial superinfections is in conflict with the recent antimicrobial stewardships in such a way that its use, although strictly related to COVID-19 patients, should be carefully evaluated under a risk/benefit light (Chakraborti et al., 2020).

Likewise, secondary bacterial infections in hospitalized COVID-19 patients with pneumonia are infrequent due to frequent empirical antibiotic treatment (Bhowmik et al., 2020). Langford et al. (2020) found that SARS-CoV-2 is often linked to Gram-positive and Gram-negative bacterial infections, similar to atypical bacteria that can cause bacterial pneumonia, ultimately increasing the risk of mortality and complications. Thus, TCs might be effective in the management of COVID-19 pneumonia due to their experimental anti-SARS-CoV-2 effects and broadspectrum antibacterial activity (Mirzaei et al., 2020).

\section{TETRACYCLINES AND COVID-19 COMPLICATIONS}

Most SARS-CoV-2 infections are mild; however, in severe cases, they are associated with systemic complications due to propagation of cytokine storm, dysregulation of the renin-angiotensin NLRP3 system (RAS), and activation of Nod-like receptor pyrin 3 (NLRP3) inflammasomes (Manna et al., 2020). The activation of NLRP3 inflammasomes and toll-like receptor 4 (TLR4) by SARS-CoV-2 or direct SARSCoV-2 invasion through ACE2 receptors, which are expressed in different tissues, may lead to various organ injuries (Polidoro et al., 2020). Among such SARS-CoV-2 infection-derived complications, acute cardiac injury, and neurological, hematological, endocrinological, and metabolic complications appear to be the most frequently observed and are collectively called as extrapulmonary manifestations of COVID-19 (Van den Berg and Te Velde, 2020).
The most common COVID-19 complications are ARDS and acute cardiac injury, although they can be mitigated by TC therapy. Gupta et al. (2020), in an experimental mice model, showed that tetracycline reduces the risk of ALI and ARDS by inhibition of NLRP3 inflammasomes. Moreover, doxycycline inhibits MMP2-mediated degradation of myosin light chain 1 and troponin during experimental myocardial ischemicreperfusion injury (Bode et al., 2019). Besides, minocycline has a cardioprotective effect during myocardial ischemic-reperfusion injury through the inhibition of NFKB activation and proinflammatory cytokine release (Bil-Lula et al., 2018). Hence, through their anti-inflammatory and immunomodulatory effects, TCs lead to a reduction of both systemic and lifethreatening complications of COVID-19 (Yi et al., 2019). Also, doxycycline and other type of TCs may reduce sepsis-induced cytokine storm and associated systemic organ damage during severe secondary bacterial infection in COVID-19 patients (Sargiacomo et al., 2020). Therefore, the net pleiotropic effect of tetracycline is summarized in Figure 1.

Nonetheless, also worthy of note is that the primary concern while using TCs in COVID-19 management is related to their contraindications in children, sulfa drug allergy, pregnancy, and lactation. Indeed, serious side effects, such as photosensitivity, drug-induced hepatitis, and erythema multiforme, may occur and thus should be evaluated prior to the use of TCs (Patel et al., 2020). In addition, COVID-19 may be linked to skin manifestations such as red itchy patches and itchy blisters that result from direct SARS-CoV-2 invasion, inflammatory reactions, and allergic reactions to repurposing drugs, such as TCs (Hamblin and Abrahamse, 2019).

\section{CONCLUSION}

Taken together, data presented here highlight that the antiviral, anti-inflammatory, immune-modulatory, and cardioprotective abilities of TCs, mainly doxycycline or minocycline, make them potential partners in the management of COVID19-derived pneumonia and related complications, such as ALI and ARDS. In addition, both doxycycline and minocycline have noteworthy therapeutic and prophylactic effects against SARSCoV-2 infection.

\section{AUTHOR CONTRIBUTIONS}

All authors listed have made a substantial, direct, and intellectual contribution to the work and approved it for publication.

\section{ACKNOWLEDGMENTS}

The authors thank all members of the College of Medicine, AlMustansiyria University. NC-M acknowledges the Portuguese Foundation for Science and Technology under the Horizon 2020 Program (PTDC/PSI-GER/28076/2017). 


\section{REFERENCES}

Al-Kuraishy, H. M., Al-Gareeb, A. I., Al-Niemi, M. S., Al-Buhadily, A. K., AlHarchan, N. A., and Lugnier, C. (2020a). COVID-19 and Phosphodiesterase Enzyme Type 5 Inhibitors. J. Microsc. Ultrastruct. 8 (4), 141-145. doi:10.4103/ JMAU.JMAU_63_20

Al-kuraishy, H. M., Al-Maiahy, T. J., Al-Gareeb, A. I., Musa, R. A., and Ali, Z. H. (2020b). COVID-19 Pneumonia in an Iraqi Pregnant Woman With Preterm Delivery. Asian Pac. J. Reprod. 9 (3), 156-158. doi:10.4103/2305-0500.282984

Alam, M. M., Mahmud, S., Rahman, M. M., Simpson, J., Aggarwal, S., and Ahmed, Z. (2020). Clinical Outcomes of Early Treatment With Doxycycline for 89 High-Risk COVID-19 Patients in Long-Term Care Facilities in New York. Cureus 12 (8), e9658. doi:10.7759/cureus.9658

Bharadwaj, S., Lee, K. E., Dwivedi, V. D., and Kang, S. G. (2020). Computational Insights into Tetracyclines as Inhibitors against SARS-CoV-2 Mpro via Combinatorial Molecular Simulation Calculations. Life Sci. 257, 118080. doi:10.1016/j.lfs.2020.118080

Bhowmik, D., Nandi, R., Jagadeesan, R., Kumar, N., Prakash, A., and Kumar, D. (2020). Identification of Potential Inhibitors against SARS-CoV-2 by Targeting Proteins Responsible for Envelope Formation and Virion Assembly Using Docking Based Virtual Screening, and Pharmacokinetics Approaches. Infect. Genet. Evol. 84, 104451. doi:10.1016/j.meegid.2020.104451

Bil-Lula, I., Krzywonos-Zawadzka, A., Sawicka, J., Bialy, D., Wawrzynska, M., Wozniak, M., et al. (2018). L-NAME Improves Doxycycline and ML-7 Cardioprotection from Oxidative Stress. Front Biosci. (Landmark Ed.) 23, 298-309. doi:10.2741/4592

Bode, C., Peukert, K., Schewe, J. C., Putensen, C., Latz, E., and Steinhagen, F. (2019). "Tetracycline Alleviates Acute Lung Injury by Inhibition of NLRP3 Inflammasome," in ERS International Congress 2019 abstracts, Madrid, Spain 28 September-02 October 2019.

Bonzano, C., Borroni, D., Lancia, A., and Bonzano, E. (2020). Doxycycline: From Ocular Rosacea to COVID-19 Anosmia. New Insight Into the Coronavirus Outbreak. Front Med. (Lausanne) 7, 200. doi:10.3389/fmed.2020.00200

Byrne, J. D., Shakur, R., Collins, J., Becker, S. L., Young, C. C., Boyce, H., et al. (2020). Prophylaxis with Tetracyclines in ARDS: Potential Therapy for COVID-19-Induced ARDS? medRxiv [Preprint]. Available at; https://doi. org/10.1101/2020.07.22.20154542 (Accessed July 28, 2020)

Cag, Y., Icten, S., Isik-Goren, B., Baysal, N. B., Bektas, B., Selvi, E., et al. (2020). A Novel Approach to Managing COVID-19 Patients; Results of Lopinavir Plus Doxycycline Cohort. Eur. J. Clin. Microbiol. Infect. Dis. 13, 1-5. doi:10.1007/ s10096-020-04099-w

Carcaterra, M., and Caruso, C. (2021). Alveolar Epithelial Cell Type II as Main Target of SARS-CoV-2 Virus and COVID-19 Development via NF-Kb Pathway Deregulation: A Physio-Pathological Theory. Med. Hypotheses 146, 110412. doi:10.1016/j.mehy.2020.110412

Chakraborti, S., Bheemireddy, S., and Srinivasan, N. (2020). Repurposing Drugs against Main Protease of SARS-CoV-2: Mechanism Based Insights Supported by Available Laboratory and Clinical Data. Mol. Omics 16. 474-491. doi:10. 1039/D0MO00057D

Conforti, C., Giuffrida, R., Zalaudek, I., and Di Meo, N. (2020). Doxycycline, a Widely Used Antibiotic in Dermatology with a Possible Anti-inflammatory Action against IL-6 in COVID-19 Outbreak. Dermatol. Ther. 33 (4), e13437. doi:10.1111/dth.13437

Connors, J. M., and Levy, J. H. (2020). Thromboinflammation and the Hypercoagulability of COVID-19. J. Thromb. Haemost. 18 (7), 1559-1561. doi:10.1111/jth.14849

Di Caprio, R., Lembo, S., Di Costanzo, L., Balato, A., and Monfrecola, G. (2015). Anti-inflammatory Properties of Low and High Doxycycline Doses: an In Vitro Study. Mediators Inflamm. 2015, 329418. doi:10.1155/2015/ 329418

Diana, G., Strollo, R., Diana, D., Strollo, M., Galassi, A. R., and Crea, F. (2020). Cardiac Safety and Potential Efficacy: Two Reasons for Considering Minocycline in Place of Azithromycin in COVID-19 Management. Euro. Heart J -Cardiovas. Pharmacothe. [Preprint] pvaa049. doi:10.1093/ehjcvp/pvaa049

Ferreira, A. C., Reis, P. A., de Freitas, C. S., Sacramento, C. Q., Villas Bôas Hoelz, L., Bastos, M. M., et al. (2019). Beyond Members of the Flaviviridae Family,
Sofosbuvir Also Inhibits Chikungunya Virus Replication. Antimicrob. Agents Chemother. 63 (2). e01389-18. doi:10.1128/AAC.01389-18

Ferreira, A. O., Polonini, H. C., and Dijkers, E. C. (2020). Postulated Adjuvant Therapeutic Strategies for COVID-19. J. Pers. Med. 10 (3), 80. doi:10.3390/ jpm10030080

Forster, P., Forster, L., Renfrew, C., and Forster, M. (2020). Phylogenetic Network Analysis of SARS-CoV-2 Genomes. Proc. Nat. Acad. Sci. U.S.A. 117 (17), 9241-9243. doi:10.1073/pnas.2004999117

Gatti, M., Fusaroli, M., Caraceni, P., Poluzzi, E., De Ponti, F., and Raschi, E. (2020). Serious Adverse Events With Tocilizumab: Pharmacovigilance as an Aid to Prioritize Monitoring in COVID-19. British J. Clin. Pharmacol. 87 (3), 1533-1540. doi:10.1111/bcp.14459

Gautam, S. S., Gautam, C. S., Garg, V. K., and Singh, H. (2020). Combining Hydroxychloroquine and Minocycline: Potential Role in Moderate to Severe COVID-19 Infection. Expert Rev. Clin. Pharmacol. 13 (11), 1183-1190. doi:10. 1080/17512433.2020.1832889

Gendrot, M., Andreani, J., Jardot, P., Hutter, S., Delandre, O., Boxberger, M., et al. (2020). In vitro antiviral Activity of Doxycycline Against SARS-CoV-2. Molecules 25 (21), 5064. doi:10.3390/molecules25215064

Guaraldi, G., Meschiari, M., Cozzi-Lepri, A., Milic, J., Tonelli, R., Menozzi, M., et al. (2020). Tocilizumab in Patients With Severe COVID-19: a Retrospective Cohort Study. Lancet Rheumatol. 2 (8), e474-e84. doi:10.1016/S26659913(20)30173-9

Gupta, A., Madhavan, M. V., Sehgal, K., Nair, N., Mahajan, S., Sehrawat, T. S., et al. (2020). Extrapulmonary Manifestations of COVID-19. Nat. Med. 26 (7), 1017-1032. doi:10.1038/s41591-020-0968-3

Hamblin, M. R., and Abrahamse, H. (2019). Tetracyclines: Light-Activated Antibiotics? Future Med. Chem. 11 (18), 2427-2445. doi:10.4155/fmc-20180513

Hariharan, A., Hakeem, A. R., Radhakrishnan, S., Reddy, M. S., and Rela, M. (2020). The Role and Therapeutic Potential of NF-Kappa-B Pathway in Severe COVID-19 Patients. Inflammopharmacology 29 (1), 91-100. doi:10.1007/ s10787-020-00773-9

Huang, A. C-C., Huang, C-G., Yang, C-T., and Hu, H-C. (2020). Concomitant Infection With COVID-19 and Mycoplasma pneumoniae. Biomed. J. 43 (5), 458-461. doi:10.1016/j.bj.2020.07.002

Kircheis, R., Haasbach, E., Lueftenegger, D., Heyken, W. T., Ocker, M., and Planz, O. (2020). NF- $\kappa B$ Pathway as a Potential Target for Treatment of Critical Stage COVID-19 Patients. Front Immunol. 11, 598444. doi:10.3389/fimmu.2020. 598444

Langford, B. J., So, M., Raybardhan, S., Leung, V., Westwood, D., MacFadden, D. R., et al. (2020). Bacterial Co-Infection and Secondary Infection in Patients With COVID-19: a Living Rapid Review and MetaAnalysis. Clin. Microbiol. Infect. 26 (12), 1622-1629. doi:10.1016/j.cmi. 2020.07.016

Li, Y., Wu, Z., Liu, K., Qi, P., Xu, J., Wei, J., et al. (2017). Doxycycline Enhances Adsorption and Inhibits Early-Stage Replication of Porcine Reproductive and Respiratory Syndrome Virus In Vitro. FEMS Microbiol. Lett. 364 (17), 364. doi:10.1093/femsle/fnx170

Luo, P., Liu, Y., Qiu, L., Liu, X., Liu, D., and Li, J. (2020). Tocilizumab Treatment in COVID-19: A Single Center Experience. J. Med. Virol. 92 (7), 814-818. doi:10. 1002/jmv.25801

Manna, S., Baindara, P., and Mandal, S. M. (2020). Molecular Pathogenesis of Secondary Bacterial Infection Associated to Viral Infections Including SARSCoV-2. J. Infect. Public Health 13 (10), 1397-1404. doi:10.1016/j.jiph.2020. 07.003

McGonagle, D., Sharif, K., O'Regan, A., and Bridgewood, C. (2020). Interleukin-6 Use in COVID-19 Pneumonia Related Macrophage Activation Syndrome. Autoimmun. Rev. 19 (6), 102537. doi:10.1016/j. autrev.2020.102537

McKenna, M. (2020). The Antibiotic Paradox: Why Companies Can't Afford to Create Life-Saving Drugs. Nature 584 (7821), 338-341. doi:10.1038/d41586020-02418-x

Mirzaei, R., Goodarzi, P., Asadi, M., Soltani, A., Aljanabi, H. A. A., Jeda, A. S., et al. (2020). Bacterial Co-Infections With SARS-CoV-2. IUBMB life 72 (10), 2097-2111. doi:10.1002/iub.2356

Mishra, M. K., and Basu, A. (2008). Minocycline Neuroprotects, Reduces Microglial Activation, Inhibits Caspase 3 Induction, and Viral Replication 
Following Japanese Encephalitis. J. Neurochem. 105 (5), 1582-1595. doi:10. 1111/j.1471-4159.2008.05238.x

Mosquera-Sulbaran, J. A., and Hernández-Fonseca, H. (2021). Tetracycline and Viruses: a Possible Treatment for COVID-19? Arch. Virol. 166, 1-7. doi:10. 1007/s00705-020-04860-8

Mostafa, M. A. (2020). Doxycycline and Minocycline Drugs as a Treatment Proposal for Inhibition of ARDS and Inflammatory Cytokine Mediators Caused by COVID19. AIJR Preprints. doi:10.21608/AUJV.2020.106725

Moullan, N., Mouchiroud, L., Wang, X., Ryu, D., Williams, E. G., Mottis, A., et al. (2015). Tetracyclines Disturb Mitochondrial Function across Eukaryotic Models: a Call for Caution in Biomedical Research. Cell Rep. 10 (10), 1681-1691. doi:10.1016/j.celrep.2015.02.034

Neufeldt, C. J., Cerikan, B., Cortese, M., Frankish, J., Lee, J. Y., Plociennikowska, A., et al. (2020). SARS-CoV-2 Infection Induces a Pro-Inflammatory Cytokine Response Through cGAS-STING and NF-kB. bioRxivAvailable at: https:// www.biorxiv.org/content/10.1101/2020.07.21.212639v1 (Accessed July 21, 2020).

Ohe, M., Furuya, K., and Goudarzi, H. (2020). Tetracycline Plus Macrolide: A Potential Therapeutic Regimen for COVID-19? BioSci. Tren. 14 (6), 467-468. doi:10.5582/bst.2020.03443

Oliveira, A. C., Richards, E. M., Karas, M. M., Pepine, C. J., and Raizada, M. K. (2020). Would Repurposing Minocycline Alleviate Neurologic Manifestations of COVID-19? Front Neurosci. 14, 577780. doi:10.3389/ fnins.2020.577780

Patel, A., Khande, H., Periasamy, H., and Mokale, S. (2020). Immunomodulatory Effect of Doxycycline Ameliorates Systemic and Pulmonary Inflammation in a Murine Polymicrobial Sepsis Model. Inflammation. 43, 1-9. doi:10.1007/ s10753-020-01188-y

Peiris, C., Gunatilake, S. R., Mlsna, T. E., Mohan, D., and Vithanage, M. (2017). Biochar Based Removal of Antibiotic Sulfonamides and Tetracyclines in Aquatic Environments: a Critical Review. Bioresour. Technol. 246, 150-159. doi:10.1016/j.biortech.2017.07.150

Poinas, A., Boutoille, D., Vrignaud, F., Nguyen, J. M., Bonnet, F., Rat, C., et al. (2020). Impact of Doxycycline on COVID-19 Patients With Risk Factors of Disease Degradation: Dynamic, A Randomised Controlled Double-Blind Trial. [Preprint from Research Square] doi:10.21203/rs.3. rs-29729/v1

Polidoro, R. B., Hagan, R. S., de Santis Santiago, R., and Schmidt, N. W. (2020). Overview: Systemic Inflammatory Response Derived from Lung Injury Caused by SARS-CoV-2 Infection Explains Severe Outcomes in COVID-19. Front Immunol. 11, 1626. doi:10.3389/fimmu.2020.01626

Portnoy, J., Waller, M., and Elliott, T. (2020). Telemedicine in the Era of COVID19. J. Allergy Clin. Immunol. Pract. 8 (5), 1489-1491. doi:10.1016/j.jaip.2020. 03.008

Sargiacomo, C., Sotgia, F., and Lisanti, M. P. (2020). COVID-19 and Chronological Aging: Senolytics and Other Anti-aging Drugs for the Treatment or Prevention of Corona Virus Infection? Aging (Albany N. Y.) 12 (8), 6511. doi:10.18632/ aging.103001

Ser, H. L., Tan, L. T., Law, J. W., Letchumanan, V., Ab Mutalib, N. S., and Lee, L. H. (2020). Genomic Analysis of Severe Acute Respiratory Syndrome Coronavirus 2 (SARS-CoV-2) Strains Isolated in Malaysia. Prog. Microbes Mol. Biol. 3 (1) 1-8.
Singh, H., Kakkar, A. K., and Chauhan, P. (2020). Repurposing Minocycline for COVID-19 Management: Mechanisms, Opportunities, and Challenges. Expert Rev. Anti Infect. Ther. 18 (10), 997-1003. doi:10.1080/14787210.2020.1782190

Sodhi, M., and Etminan, M. (2020). Therapeutic Potential for Tetracyclines in the Treatment of COVID-19. Pharmacotherapy. J. Hum. Pharmacol. Drug Ther. 40 (5), 487-488. doi:10.1002/phar.2395

Stone, J. H., Frigault, M. J., Serling-Boyd, N. J., Fernandes, A. D., Harvey, L., Foulkes, A. S., et al. (2020). Efficacy of Tocilizumab in Patients Hospitalized with COVID-19. New Engl. J. Med. 383 (24), 2333-2344. doi:10.1056/ NEJMoa2028836

Sun, J., Shigemi, H., Tanaka, Y., Yamauchi, T., Ueda, T., and Iwasaki, H. (2015). Tetracyclines Downregulate the Production of LPS-Induced Cytokines and Chemokines in THP-1 Cells via ERK, P38, and Nuclear Factor-kB Signaling Pathways. Biochem. Biophys. Rep. 4, 397-404. doi:10.1016/j.bbrep.2015. 11.003

Trivedi, N., Verma, A., and Kumar, D. (2020). Possible Treatment and Strategies for COVID-19: Review and Assessment. Eur. Rev. Med. Pharmacol. Sci. 24 (23), 12593-12608. doi:10.26355/eurrev_202012_24057

Ueland, T., Holter, J. C., Holten, A. R., Müller, K. E., Lind, A., Bekken, G. K., et al. (2020). Distinct and Early Increase in Circulating MMP-9 in COVID-19 Patients with Respiratory Failure. J. Infect. 81 (3), e41-e43. doi:10.1016/j. jinf.2020.06.061

Van den Berg, D. F., and Te Velde, A. A. (2020). Severe COVID-19: NLRP3 Inflammasome Dysregulated. Front Immunol. 11, 1580. doi:10.3389/fimmu. 2020.01580

Wang, D., Hu, B., Hu, C., Zhu, F., Liu, X., Zhang, J., et al. (2020). Clinical Characteristics of 138 Hospitalized Patients With 2019 Novel CoronavirusInfected Pneumonia in Wuhan, China. JAMA 323 (11), 1061-1069. doi:10. 1001/jama.2020.1585

Yates, P. A., Newman, S. A., Oshry, L. J., Glassman, R. H., Leone, A. M., and Reichel, E. (2020). Doxycycline Treatment of High-Risk COVID-19-Positive Patients with Comorbid Pulmonary Disease. Ther. Adv. Respir. Dis. 14, 1753466620951053. doi:10.1177/1753466620951053

Yi, Q., Tan, F. H., Tan, J. A., Chen, X. H., Xiao, Q., Liu, Y. H., et al. (2019). Minocycline Protects against Myocardial Ischemia/Reperfusion Injury in Rats by Upregulating MCPIP1 to Inhibit NF-kB Activation. Acta Pharmacol. Sin. 40 (8), 1019-1028. doi:10.1038/s41401-019-0214-z

Zhao, T. Y., and Patankar, N. A. (2020). Tetracycline as an Inhibitor to the Coronavirus SARS-CoV-2. arXiv [Preprint] Available at: arXiv:2008.06034 (Accessed August 13, 2020).

Conflict of Interest: The authors declare that the research was conducted in the absence of any commercial or financial relationships that could be construed as a potential conflict of interest.

Copyright (ㅇ 2021 Al-kuraishy, Al-Gareeb, Alqarni, Cruz-Martins and El-Saber Batiha. This is an open-access article distributed under the terms of the Creative Commons Attribution License (CC BY). The use, distribution or reproduction in other forums is permitted, provided the original author(s) and the copyright owner(s) are credited and that the original publication in this journal is cited, in accordance with accepted academic practice. No use, distribution or reproduction is permitted which does not comply with these terms. 